\title{
Oral health-related attitudes and behaviours of drug users
}

Drug users: oral health-related attitudes and behaviours

\section{P. G. Robinson, S. Acquah and B. Gibson Br Dent J 2005; 198: 219-224}

Aim

Explore oral health-related attitudes and behaviours of drug users.

Design

Qualitative study using focus groups and semi-structured interviews.

Setting

Facilities for treatment and recovery of drug users in South London.

Participants

Twenty-six male and 14 female recovering drug users.

Results

Participants described many changes in lifestyle associated with drug use including the physical effects of drugs, dietary habits, organisational and time constraints and unfavourable social conditions, which were not conducive to oral health. There was considerable health consciousness. They associated general health problems and problems with teeth, gums and oral soft tissues to both direct and indirect effects of drug use. Use of dental services was inhibited by low priority for oral health relative to the need to obtain and use drugs, experientially induced fear of dentists, the acceptability of dental services, needle-phobia, ability to self-medicate and organisational factors in their lifestyles.

\section{Conclusions}

The lifestyles of drug users may contribute to oral health problems and low use of services. Drug users therefore comprise a group with special dental needs and need greater access to dental care than most people. Much of this care could be provided in general practice where appropriate dental care can contribute to recovery from drug use.

\section{IN BRIEF}

- Drug users have a special need for dental care.

- Oral health can be a low priority for them whilst using drugs.

- Drug users have the same entitlement as anyone else to dental care.

- Barriers to care include fear of dentists, needle phobia, the acceptability of dental services, the ability to self-medicate and organisational factors in their lifestyles.

- Dental care of recovering drug users can not only improve their oral health, but can assist them construct a 'non-addict' identity.

\section{COMMENT}

This paper addresses a very important area. There is little in the UK dental literature on the issue of oral health needs and oral healthcare provision for people who use intravenous drugs. These interesting results give an evidence base to previous anecdotal reports and perceptions of people working in the field.

Exploring attitudes of forty recovering drug users, it provides some explanations of the different priorities that individuals give to oral health due to their drug using lifestyle.

Barriers to oral health included homelessness, lack of hygiene facilities and tools for oral care, feeling of worthlessness and low self esteem. Elements of a drug using lifestyle such as preoccupation with obtaining drugs and avoiding withdrawal, lack of ability to attend appointments and finish courses of treatment act as barriers to care. Anxiety and needle phobia were common.

Risk factors to oral health included xerostomia, high sugar consumption and poor eating habits, poor oral hygiene, use of tobacco and breaking or losing teeth during fighting and accidents. Other problems of self care were reported including wretching and nausea while brushing teeth.

With regard to access to oral heathcare services, many reported avoiding dentists until pain became unbearable. Most had selfmedicated for dental pain and some had removed their own teeth. Some hid their drug use from dentists because they did not want to be treated differently. Other, more positive attitudes were reported including feeling comfortable with dentists if they had the right approach which indicates that training for the dental team in communication skills and management of drug users would help break down the barriers. The authors recommend that most of these individuals would be suitable for treatment in general dental practice. However, traditional patterns of registration with dentists paid on a fee per item basis are not suitable. Dentists may be unwilling to accept those they fear may not complete treatment causing them to lose valuable time and income. These problems may be overcome by new general dental service contractual arrangements or other locally planned services with the support of special care services for provision of more complex care and provision of sedation or general anaesthesia where appropriate. Treatment plans may need to be modified while maintaining patient centred care principles.

This excellent paper also emphasises the important role that oral health care plays in recovery from drug use and how a holistic approach to health will help in rehabilitation of these individuals.

D. Lewis, Senior Community Dentist, Dorset NHS Trust doi: $10.1038 /$ sj.bdj.4812088 